\title{
A NOVEL ADAPTIVE - WAVELET BASED DETECTION ALGORITHM FOR CHIPLESS RFID SYSTEM
}

\author{
Meriam A. Bibile and Nemai C. Karmakar \\ Department of Electrical and Computer Systems Engineering, \\ Monash University, Melbourne, Australia
}

\begin{abstract}
In this paper, a novel wavelet-based detection algorithm is introduced for the detection of chipless RFID tags. The chipless RFID tag has a frequency signature which is identical to itself. Here a vector network analyser is used where the received backscatter signal is analysed in frequency domain. Thus the frequency signature is decoded by comparing the wavelet coefficients which identifies the bits accurately. Further, the detection algorithm has been applied for the tag detection under different dynamic environments to check the robustness of the detection algorithm. The new method doesn't rely on calibration tags and shows robust detection under different environments and movement.
\end{abstract}

\section{KEYWORDS}

Chipless RFID, wavelet, backscatter signal, frequency domain

\section{INTRODUCTION}

The chipless RFID reader extracts the backscattered signal and decodes the tag ID. This is an ongoing challenge, as the detection procedure for a chipless RFID tag has more complexities compared to a conventional RFID tag. The signal collides with other scatterers or tags which give a 'clutter' signal with interference. A number of detection techniques have been applied to achieve an accurate result of its tag ID.

The basic detection technique is based on comparing the received data with threshold values obtained by calibration. It is, therefore, a basic approach and it does not possess the flexibility and adaptability required in the detection process to address errors due to a dynamic environment [1]. Different types of detection algorithms and decoding techniques have been revealed in the past few years.

Moving average technique is a simple de-noising technique which removes noises by acting as a low pass filter. An 11 sample averaging moving average filtering has been successfully implemented on a low-cost mid-range microcontroller having low processing power capabilities, and a smoothened waveform is resulted after using this filtering technique. Hilbert transform Dhinaharan Nagamalai et al. (Eds) : CoSIT, SIGL, AIAPP, CYBI, CRIS, SEC, DMA - 2017

pp. 29-38, 2017. @ CS \& IT-CSCP 2017

DOI : $10.5121 /$ csit.2017.70404 
(HT) is a complex analytical signal processing technique [2]. This technique has been used to reconstruct the frequency signatures of the chipless tags. It has been experimentally proven that HT provides the extraction of the amplitude and phase functions of the frequency signature.

The signal space representation of chipless RFID tags uses an efficient mathematical model to decode information in a chipless RFID tag [3-4]. The frequency signatures are represented by a matrix which is composed of orthonormal column vectors and a singular value matrix. The constellation of signal points are plotted with a basis function. It can be seen that as the number of bits increase this method will face limitations. Matrix pencil method (MPM) and Short time matrix principle method (STMPM) are two more detection techniques that have been applied for chipless RFID systems [5-6]. These two techniques are applied in the time domain and are mentioned as accurate detection techniques in extracting the carrier to noise ratio (CNR) of the response. Detection is performed by extracting the poles and residues from the backscattered signal using the Matrix Pencil Algorithm. A Maximum Likelihood (ML) based tag detection technique and Trellis decoding technique has been developed where detection error rate is compared with the bit to bit detection [7]. It has been found that ML detection has the best performance. It also reports that the computational complexity is higher in ML detection technique than Trellis detection technique.

The main aim of this paper is to develop a detection algorithm which can be practically applied in firmware. As many of the above algorithms are highly complexed in implementing in the chipless RFID reader. Also, most of them have the limitation in the number of bits that can be detected. In this paper, we have developed a novel wavelet which suits the chipless RFID received signal to detect the frequency ID of the chipless RFID tag.

\section{SYSTEM MODEL AND DESIGN}

In this section the system model of the chipless RFID system used in this analysis is discussed. A description of the backscattered signal is also given and the backscattered signal of the used tag is analysed using simulation results obtained through CST microwave studio.

\subsection{Experimental Model}

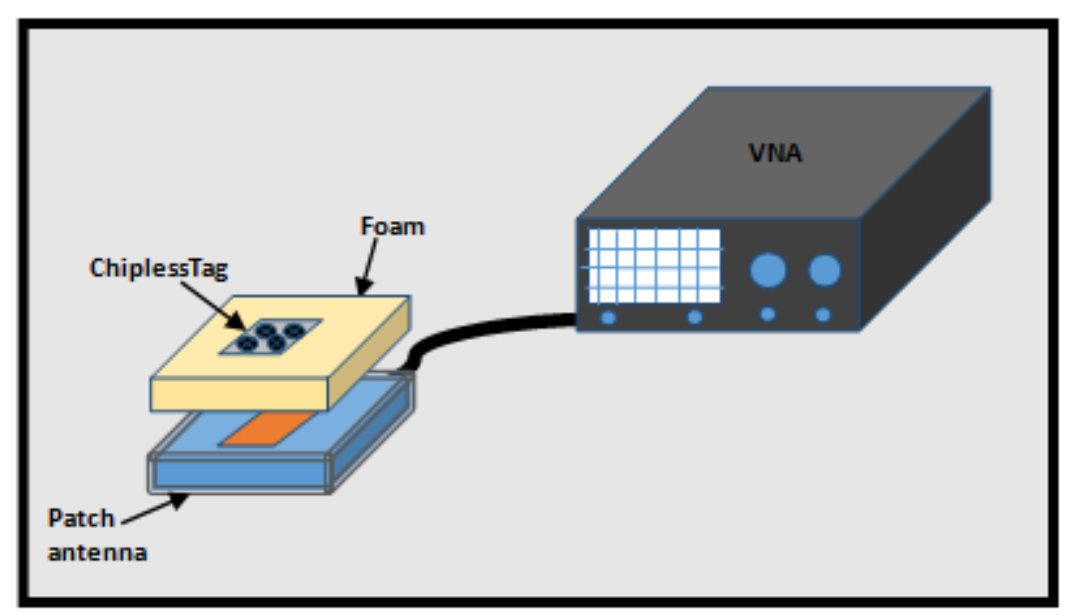

Figure 1-Chipless RFID system 
The experiment is performed using a 5-bit spiral resonator design. Each resonator has its own resonance frequency and has a diameter of $5 \mathrm{~mm}$. The tag with 5 bits has a dimension of $2 \mathrm{~cm} \times$ $2 \mathrm{~cm}$. The tag design is not presented in this paper to due to the confidentiality of the tag design. The tag RCS is shown in Fig 2. A patch antenna is used for the measurements, and the reading is taken by loading the antenna with a tag using vector network analyzer (VNA) as shown in Fig 1. The tag is loaded above the patch antenna and is placed on a piece of foam with $1 \mathrm{~cm}$ thickness.

Backscattered signals from chipless RFID tags are very weak, and the detection process at the RFID reader is extremely susceptible to noise. This is because the information is represented as analog variations as opposed to a modulated digital stream of data as in conventional wireless communication of information where error correction schemes can be used to detect the presence of errors and discard erroneous data packets.

According to Fig 2, the resonant frequencies of the tag are located at 4.15, 4.70, 5.37, 6.10 and $6.95 \mathrm{GHz}$ respectively. The other local maxima detected in the signal are due to the amplitude spectrum of the background and antenna S11. These spurious peaks need to be carefully filtered to detect the correct frequency signature of the chipless RFID tag.

\section{Tag RCS vs. Frequenncy}

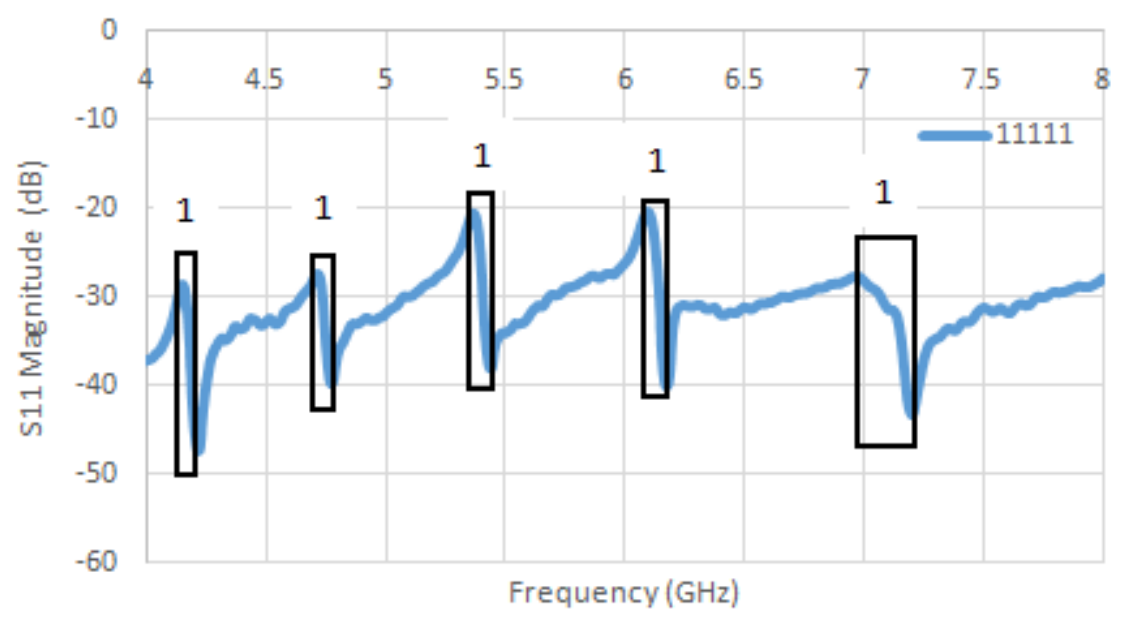

Figure 2 - S11 Magnitude (dB) vs. frequency of the 5-bit resonant tag

\subsection{Wavelet Design}

In this paper, a novel wavelet is adopted to detect the peaks of the backscattered signal. It is based on the Gaussian function which is given by (1).

$$
f(x)=a \times e^{\frac{-(x-b)^{2}}{2 c^{2}}}
$$

where $a$ is the height, $b$ is the position and $c$ is the width of the wavelet. The values $a, b$ and $c$ are adjusted to maximize the detection.

The novel wavelet design is shown in Fig 3. The width of the wavelet is adaptive according to the number of bits in the tag. In Fig 3, the width of the wavelet has been taken as $250 \mathrm{MHz}$ giving a 
wider bandwidth to the wavelet. Further for the detection of the results this has been changed to $100 \mathrm{MHz}$ giving better resolution of the detected bits. This is an advantage of this algorithm as higher number of bits can be detected with better resolution.

The detected signal is compared with the wavelet, and a coefficient is defined to represent how closely correlated the wavelet is with each part of the signal. The larger the coefficient is in absolute value, the more the similarity appears. The wavelet is shifted until it covers the whole signal. The threshold coefficient is defined after performing the experiment for the tag with bit ' 11111 ' number of times under different environmental changes. By the variation in the detected signal under different circumstances a minimum and maximum coefficient is set giving rise to a confident band for detection.

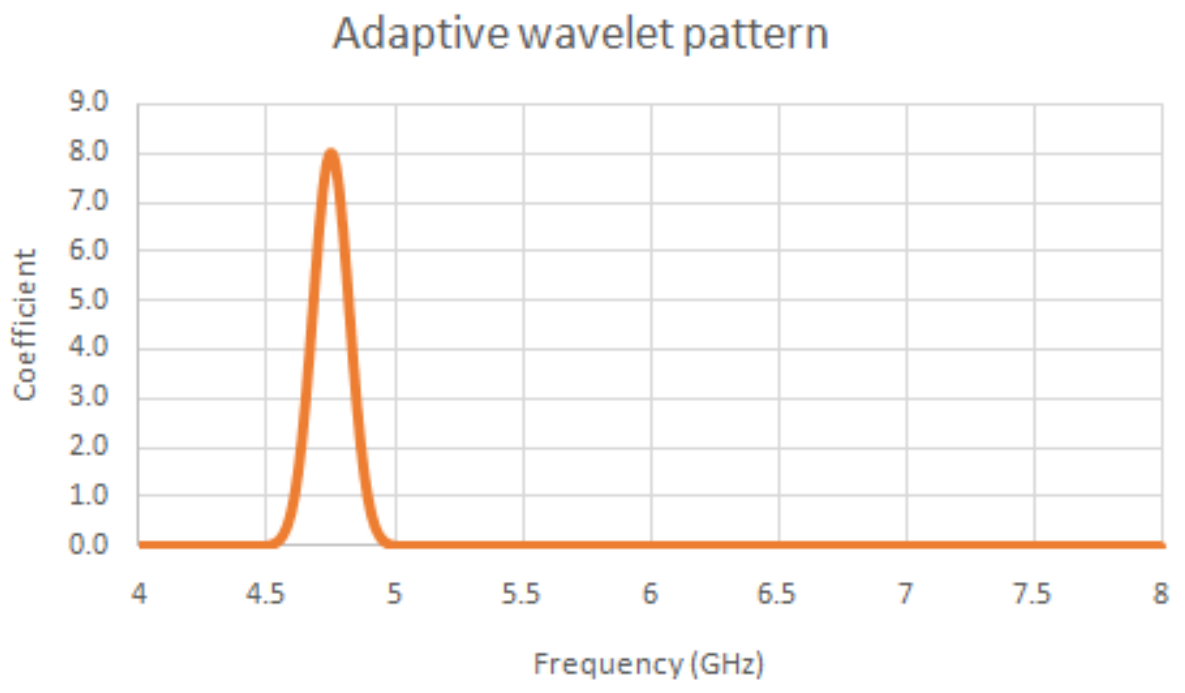

Figure 3 - New adopted wavelet pattern using Gaussian function

\subsection{Flowchart for Detection Algorithm}

The flowchart given in Fig 4, shows the steps of the developed algorithm and how it is applied to the received signal.

First the measured results using VNA are loaded into Matlab. The post processing is performed using Matlab programming software. The program can be directly downloaded to the microcontroller of the frequency domain reader developed by the research group which will be implemented in the next step of this research.

A baseline removal is performed on the first results to remove the nulls of the antenna S11. Then the wavelet is defined and is integrated with the resulting signal. The tag ID is decoded based on the condition band defined for the detection.

Experiments have been performed under different indoor dynamic environments such as placing clutter objects above the chipless RFID tag. Fig 8, shows the placing of hand $7 \mathrm{~cm}$ above covering the tag adding back reflection to the received signal. Similarly, a copper plate was also placed above the tag at $7 \mathrm{~cm}$, and the received signal was observed using the VNA. 


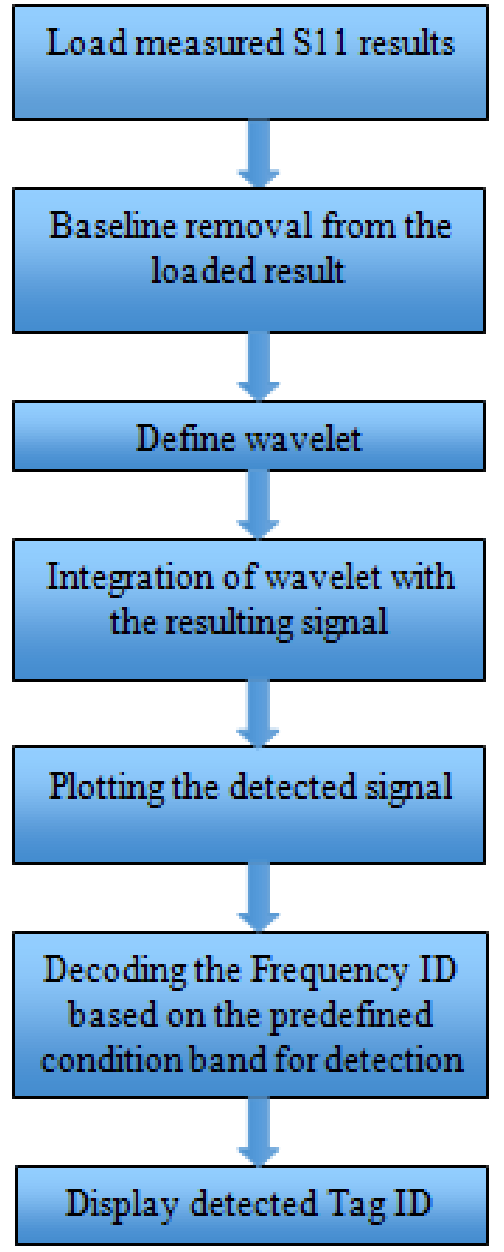

Figure 7 - Flowchart for applying the detection algorithm

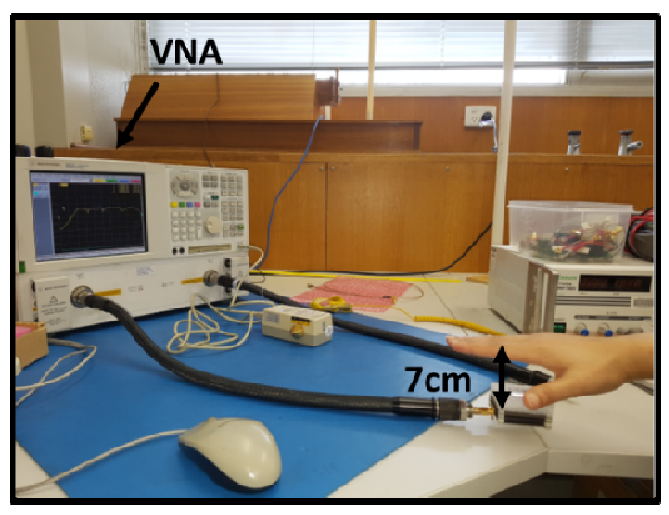

Figure 8 - placing hand on top of the tag at $7 \mathrm{~cm}$ distance giving attenuation to the signal received at the VNA 


\section{RESULTS}

The measured results of the tag detection are shown in Fig 9.

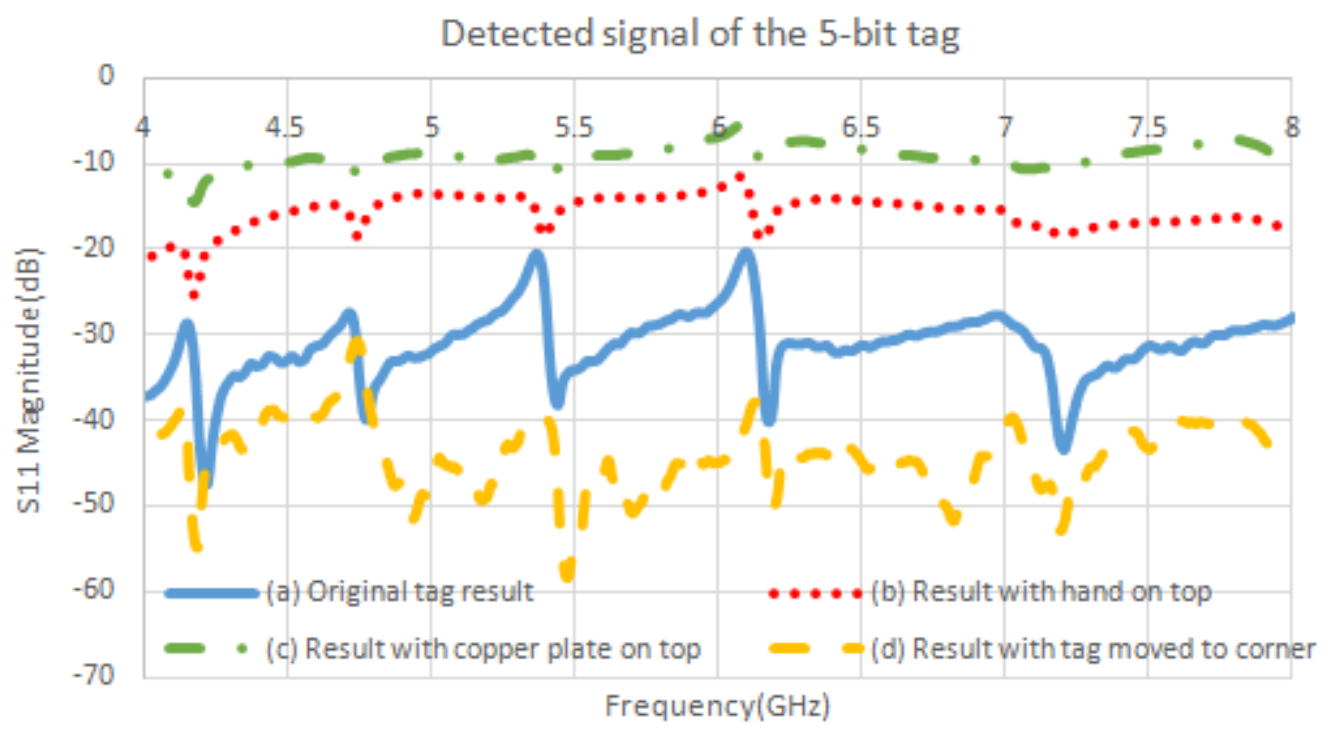

Figure 9 - Measured results (a) of the original tag at $1.5 \mathrm{~cm}$ above the patch antenna, (b) with hand placed above the tag at a distance of $7 \mathrm{~cm}$, (c) with copper plate placed on top of the tag at a distance of $7 \mathrm{~cm}$, (d)

by placing the tag at the corner of the patch antenna

It shows the received signals from the reader before applying the algorithm. In the original signal with the tag, we can identify the 5-bit resonances from the received signal itself. But when the hand, the copper plate is placed on top of the tag we can see that the received signal has increased in magnitude and has been shifted up. Also, peaks are not distinguishable at a glance. When the tag is placed in the corner of the antenna which implies the tag is moved out of the beam width of the antenna, we can again see a distortion in the signal as well as shifting the signal down in magnitude.

\section{ANALYSIS}

The detected signal after applying the detection algorithm is analysed in this section. The data is then interpreted to the end user. The results obtained after applying the developed wavelet based detection algorithm is given in Fig 10. 


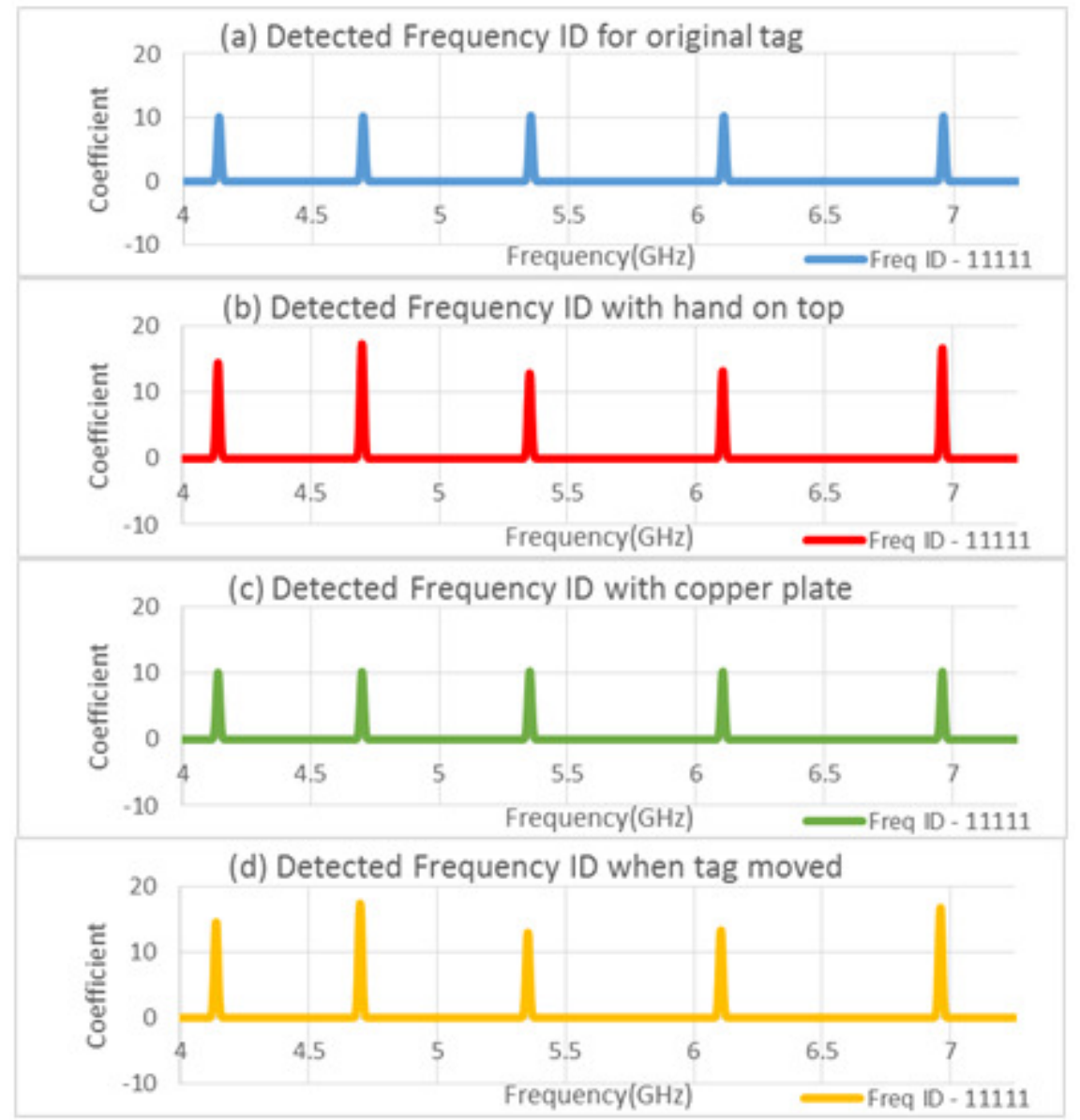

Figure 10 - Wavelet coefficients of the detected signals after applying the developed algorithm. (a) Original tag detection (b) Hand on top of tag (c) Copper plate on top of tag (d) Tag moved to the corner of antenna

The coefficients obtained are analysed in Table 1 . The coefficients of the original tag are set to 10 which is taken as the threshold value of detection for the original tag with bits ' 11111 '. A condition band is set for the maximum and minimum detectable coefficient bands. For the chipless RFID tag used in this experiment, the condition band is set between \pm 8 , which gives +18 as the maximum value of detection as bit ' 1 ' and +2 as the minimum level of detection as bit ' 1 '. If the coefficient is below or above these values, it will detect its ID as a bit ' 0 '.

When the hand is placed, we can see that the coefficients are varying and the maximum coefficient reaches 17.35. When the copper plate is placed, it reaches a maximum value of 10.32 and the tag moved to the corner of the antenna gives a coefficient of 17.35. According to these results, the frequency ID of the tag is detectable as '11111' under all the applied conditions. 
Table 1 - Wavelet coefficients for (a) Original tag detection - when the antenna is loaded with tag only (b) Hand on top of tag - when the hand is placed at $5 \mathrm{~cm}$ distance above the tag (c) Copper plate on top of tag when a copper plate of dimensions $10 \times 10 \times 0.2 \mathrm{~mm}$ is placed at $5 \mathrm{~cm}$ distance above the tag (d) Tag moved to the corner of antenna - when the tag is placed in the corner of the antenna

\begin{tabular}{|c|c|c|c|c|}
\hline $\begin{array}{c}\text { Resonance } \\
\text { Freq. } \\
(\mathrm{GHz})\end{array}$ & $\begin{array}{c}\text { Original tag } \\
\text { detection } \\
\text { (Coefficient value) }\end{array}$ & $\begin{array}{c}\text { Hand on top } \\
\text { of tag }\end{array}$ & $\begin{array}{c}\text { Copper plate on } \\
\text { top of tag }\end{array}$ & $\begin{array}{c}\text { Tag moved to } \\
\text { corner of } \\
\text { antenna }\end{array}$ \\
\hline 4.15 & 10.14 & 14.53 & 10.15 & 14.53 \\
\hline 4.70 & 10.25 & 17.35 & 10.25 & 17.35 \\
\hline 5.37 & 10.32 & 12.88 & 10.32 & 12.88 \\
\hline 6.10 & 10.29 & 13.26 & 10.29 & 13.26 \\
\hline 6.95 & 10.27 & 16.72 & 10.27 & 16.72 \\
\hline
\end{tabular}

The frequency domain reader developed in MMARS lab uses the same patch antenna design for detection and is working in the frequency range of $4-7.5 \mathrm{GHz}$. Therefore future direction of this research will be the implementation of the novel detection algorithm in firmware.

\section{CONCLUSIONS}

According to these results, it can be seen that a hand or conductive material $(\mathrm{Cu})$ placed in the close vicinity of the tag (less than $10 \mathrm{~cm}$ ) will have an effect on the magnitude of the tag measurement. Still applying the novel detection algorithm the tag can be identified even if the conductive material is placed covering the tag. Also, when the tag is moved around the surface area covered by the antenna, the tag can be detected using this detection algorithm. Since the experiment was conducted by placing the clutter only on one side of the tag, more experiments should be performed by varying the position, size, material, etc. to conclude the probability of error due to the different material surrounding the tag.

An advantage of this detection algorithm is that it could detect up to a minimum of $1 \mathrm{~dB}$ magnitude variation with added noise. The adaptability of the wavelet is another advantage of this algorithm as it can be applied for any type of tag in time domain or frequency domain and could cover the required frequency range. Also the detected bit resolution is high, which leaves room for the applications with higher number of bits in future.

When compared with other algorithms this has the most adaptability to any kind of chipless RFID system. Comparing the number of bits of a tag, the presented algorithm can be applied for higher number of bits whereas algorithms developed by Kalansuriya[3], Rezaiesarlak [6] and Divarathna[7] are implemented for low number of bits due to their mathematical complexity. As the width of the wavelet used in the detection is only $10 \mathrm{MHz}$, with a guard band of another $10 \mathrm{MHz}$ a $20 \mathrm{bit}$ tag can be easily detected in the given frequency range of $4-8 \mathrm{GHz}$. 
The algorithm can be adapted into a wider bandwidth of frequencies and can be applied for any other chipless tag design in time or frequency domain.

Most of the developed detection algorithms in this research area are tested only for simulation results or for measured results using a vector network analyser. Presently the algorithm has been programmed into the microcontroller of the chipless RFID reader.Therefore this tag detection algorithm can be used further to investigate the robustness of the chipless RFID reader system under a dynamic environment. A further study and comparison of the detection error rate applying this algorithm will be performed based on results. It can be concluded that the simplicity of this algorithm allows it to be implemented in firmware and can be further fine-tuned to give robust detection of the tag in a real environment of chipless RFID tag detection.

\section{ACKNOWLEDGEMENTS}

This work is supported by the Australian Research Council Link Project Grant LP130101044:Discreet Reading of Printable Multi-bit Chipless RFID Tags on Polymer Banknotes.

\section{REFERENCES}

[1] R. V. Koswatta, and N. C. Karmakar, "A novel reader architecture based on UWB chirp signal interrogation for multiresonator-based chipless RFID tag reading," IEEE Transactions on Microwave Theory and Techniques, vol. 60, no. 9, pp. 2925-2933, 2012.

[2] R. V. Koswatta, and N. C. Karmakar, "Moving Average Filtering Technique for SignalProcessing in Digital Section of UWB ChiplessRFID Reader,"Proceedings of Asia-Pacific Microwave Conference, pp. 1304-1307, 2010.

[3] P. Kalansuriya, N. C. Karmakar, and E. Viterbo, "Signal space representation of chipless RFID tagfrequency signatures Signal space representation of chipless RFID tagfrequency signatures,",IEEE Globecom 2011 proceedings, vol. 68, no. 7-8, pp. 437-445, 2013.

[4] P. Kalansuriya, N. C. Karmakar, \& Viterbo, E. (2013). On the detection of chipless RFID through signal space representation. Annales des Telecommunications/Annals of Telecommunications, 68(78), 437-445. doi: 10.1007/s12243-013-0377-4

[5] R. Rezaiesarlak, \& M. Manteghi, (2013). Short-time matrix pencil method for chipless RFID detection applications. IEEE Transactions on Antennas and Propagation, 61(5), 2801-2806. doi: 10.1109/TAP.2013.2238497

[6] Rezaiesarlak, R., \& Manteghi, M. (2014). A space-frequency technique for chipless RFID tag localization. IEEE Transactions on Antennas and Propagation, 62(11), 5790-5797. doi: 10.1109/TAP.2014.2350523

[7] C. Divarathna, N.C. Karmakar, "A Maximum Likelihood Based Tag Detection Technique for MIMO Chipless RFID Systems," IEEE International Microwave and RF Conference (IMaRC), 2014.

[8] S. Preradovic, I. Balbin, N. C. Karmakar, and G. F. Swiegers, "Multiresonator-based chipless RFID system for low-cost item tracking," IEEE Trans.Microw. Theory Tech., vol. 57, no. 5, pp. 1411-1419, May 2009. 
[9] A. Lazaro, A. Ramos, D. Girbau, and R. Villarino, "Chipless UWB RFID tag detection using continuous wavelet transform," IEEE Antennas and Wireless Propag. Lett., vol. 10, pp. 520-523, May 2011.

[10] A. Blischak and M. Manteghi, "Pole residue techniques for chipless RFID detection," in Proc. IEEE Antennas Propag. Soc. Int. Symp., Jun. 2009, pp. 1-4.

\section{AUTHORS}

Meriam Anushani Bibile (M'16) received the BSc.Degree in Engineering Physics from University of Colombo, Sri Lanka, in 2004 and MSc. Degree in Mobile,Personal andSatellite Communications from University of Westminster,London, United Kingdom, in 2006. She is currently working toward the Ph.D. degree in Electrical Engineering at Monash University, Melbourne, Australia. From 2008 - 2013 she was a Lecturer in Electronics and Telecommunications at the Institute of Technology, University of Moratuwa, Sri Lanka. Her areas of interests include chipless RFID, digital signal processing and digital electronics.

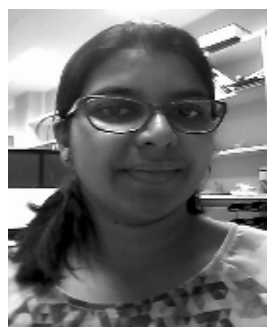

NemaiChandra Karmakar (S'91-M'91-SM'99) received the Ph.D. degree in information technology and electrical engineering from the University of Queensland, St. Lucia, Australia, in 1999. He has 20 years of teaching, design, and research experience in smart antennas, microwave active and passive circuits, and chipless RFIDs in both industry and academia in Australia, Canada, Singapore and Bangladesh. $\mathrm{He}$ has authored and co-authored more than 230 referred journal and conference papers, 24 referred book chapters and three edited and one co-authored books in the field of RFID. He has two patent applications for chipless RFIDs. Currently he is an Associate Professor with the Department of Electrical and Computer Systems

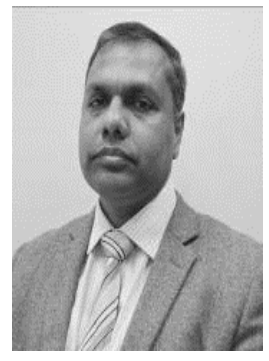
Engineering, Monash University, Melbourne, Australia. 